\title{
ZINC(II)-CHLORIDE INDUCED THIOALKYLATION OF ALUMINIUM ENOLATES; ENANTIOSELECTIVE SYNTHESIS OF ESTRADIOL-3-METHYL- 17-tert-BUTYL DIETHER
}

\author{
Ulnch Groth $^{*}$, Thomas Kohler, and Thomas Taapken \\ Institut fur Organische Chemie der Universitat Götungen, Tammannstraße 2, D-3400 \\ Gottingen, Federal Republic of Germany
}

(Recelved in Germany 29 May 1990)

\begin{abstract}
Zinc(II)-chlonde induced thoalkylation of the aluminium enolate 6 generated by conjugate reduction of the enone 5 leads - directly or via its trimethylsilylenol ether 6 - to alkylated hydrundanones $\mathbf{1 0}$ which are important intermediates in the synthesis of 19-norsteroids such as the title compound estradiol-3-methyl-17-tert-butyl diether 12
\end{abstract}

\section{Introduction}

19-Norsteroids such as estrone are attractuve target molecules in organic synthesis because of their biological activity and pharmaceutical importance ${ }^{1}$ In this communication a total synthesis of enantiomerically pure estradiol-3-methyl-17-tert-butyl diether 12 - a precursor of (+)-estrone - is described This synthesis follows the $\mathrm{CD} \rightarrow \mathrm{ACD}->$ ABCD approach Establishing the desired trans fusion of rings $C$ and $D$ and alkylation of the aluminium enolate 6 to incorporate the missing part of the seco-steroidal skeleton are the two main aims of this synthesis Recently Daniewski and Kiegiel reported ${ }^{2}$ about the diastereoselective conjugate reduction ${ }^{3}$ of the enone 5 using a tert-butylcopper(I) / HMPA / DIBAH / reagent which afforded the saturated trans-alumınum enolate 6 This enolate reacts in moderate yields only with very reactuve electrophiles such as aldehydes, acyl chlondes or allyl bromides ${ }^{4}$ In the present studies alkylation of the aluminum enolate 6 and its corresponding stlylenol ether 5 is performed using the thioalkylation method $\alpha$-Thiophenylcarbenium-ions formed from 
$\alpha$-chloro- $\alpha$-thıophenylalkanes with Lewis acids are very strong electrophiles and able to thioalkylate various enolates $^{6}$ and silylenol ethers 7

\section{Results and Discussion}

The zunc enolate 3 was generated by the $\mathrm{BuCu}(\mathrm{I})$ / HMPA / DIBAH - catalyzed conjugate reduction of 3-methyl-2-cyclopenten-1-one (1) and consecutive transmetallation with 2 equivalents zinc chlonde Thioalkylation of the zinc enolate 3 afforded in $67 \%$ yield an 8020 muxture of rac-4 and the corresponding racemic $c i s$ isomer In view of this result the thioalkylation of the zinc enolate from enone 5 formed by transmetallation of the aluminium enolate 6 with 2 equivalents of zinc chlonde was investigated

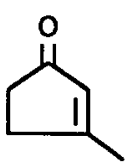

1

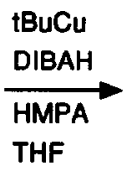

$-78^{\circ} \mathrm{C}$

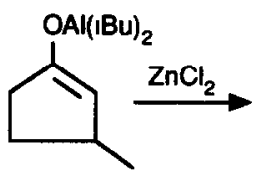

2

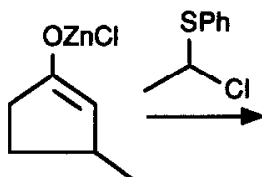

3<smiles>CC(=S)[C@H]1C(=O)CC[C@H]1C</smiles>

rac-4

The conjugate reduction of the enone 5 to the aluminum enolate 6 was performed by DIBAH in the presence of one equivalent tert-butylcopper and 8 equivalents of HMPA which were necessary as a ligand for the copper or aluminum in this reaction It turned out to be of importance to work under extreme exclusion of oxygen in order to achieve the desired products in respectable yields of about $80 \%$ with a diastereomenc excess of $>98 \%$ Otherwise the 1,2-reduction of the starting enone becomes the predominant reaction and the diastereoselectivity of this reaction decreases dramatically This can be explained by a rapid decomposition of the in situ generated reducing agent Allylic alcohol as the result of 1,2-reduction was also the man product when cosolvents other than HMPA were used Tetramethylethylenedıamıne (TMEDA), 1,3-dımethyltetrahydro-2-(1H)-pyrımıdınone (DMPU), 8 tetraethylsulfamide (TES) and tripıpendinophosphine oxıde (TPPO) instead of the toxic and carcinogenic HMPA were tried as cosolvents 


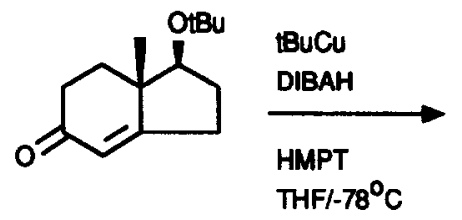

5<smiles>CCCCOC1=C[C@H]2CC[C@@H](OCCC)[C@]2(C)CC1</smiles>

1) $\mathrm{ZnCl}_{2}$

6

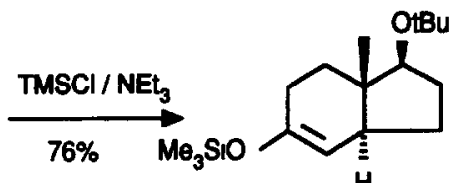

H

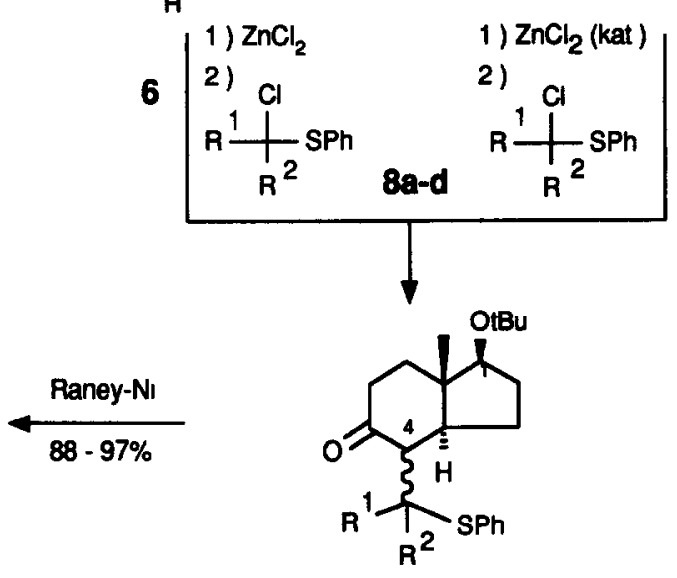

7<smiles>[R7]C([R7])[C@H]1C(=O)CC[C@@]2(C)[C@H](O)CC[C@H]12</smiles>

10a-d

\section{9a-d}

Table 1. Alkylation of the aluminum enolate 6 and the silylenol ether 7 yreld(\%)

\section{8-10 $R^{1}$}

$R^{2}$

via 6

via 7

a $\quad \mathrm{CH}_{3}$

b $\mathrm{CH}_{3}$

c $\quad \mathrm{CH}_{2} \mathrm{C}_{6} \mathrm{H}_{5}$

d $\mathrm{CH}_{2}\left(\mathrm{~m}-\mathrm{OCH}_{3}\right) \mathrm{C}_{6} \mathrm{H}_{4}$
H

$\mathrm{CH}_{3}$

$\mathrm{H}$

$\mathrm{H}$
35

71

60

56

27

65

Transmetallation of the aluminum enolate 6 with 11 equivalents of zinc chlonde afforded the corresponding zınc enolate, which was subsequently thoalkylated with the $\alpha$-chloro- $\alpha$ phenylthoalkanes 8a-d yielding the $\beta$-phenylthoalkanes 9a-d in 27-35\% yneld Due to the 11 equivalents of zinc chlonde the products 9a-d partly decomposed under these reaction conditions The large amount of zinc chlonide was necessary, because the HMPA which had to be used for the conjugate reduction of the enone 5 had already formed a complex with the zinc chloride 
To avord the presence of great amounts of inorganic salts which possibly initiated the decomposition of the $\beta$-phenylthioalkanes 9a-d, the enolate 6 was trapped as its trimethylsilylenol ether 7 Therefore trimethylsilyl chlonde was added to the reaction mixture at $-40^{\circ} \mathrm{C}$ after the conjugate reduction of 5 was completed Then the reaction mixture was allowed to warm up to room temperature After 3 hours triethylamine was added before the work-up procedure to prevent the hydrolysis of the tnmethylsilylenol ether which was 1solated in $76 \%$ yneld with $>98 \%$ de (determined by ${ }^{1} \mathrm{H}$ NMR and ${ }^{13} \mathrm{C}$ NMR spectroscopy on the signals of the 7a-methyl group at $\delta=076 \mathrm{ppm}$ and $\delta=1074 \mathrm{ppm}$ for the trans-1somer and at $\delta=1.02 \mathrm{ppm}$ and $\delta=2071 \mathrm{ppm}$ for the $c i s$-1somer)

This silylenol ether could be converted with the $\alpha$-chloro- $\alpha$-phenylthioalkanes $8 \mathrm{a}$ - $\mathrm{d}$ into the compounds 9a-d mediated by catalytic amounts of zinc chlonde in respectable yields from $56 \%$ to $71 \%$ (see Table 1) as 31 mixtures of epimers at C-4 and 11 mixtures at C-1' (determined by ${ }^{13} \mathrm{C}$ NMR spectroscopy on the signals for C-5, C-4 and C-1') Desulfurization and simultaneous equilibration of 9a-d with Raney nickel in ethanol yielded the saturated ketones 10a-d with the substituents at C-4 in equatorial position enantiomerically and diastereomencally pure

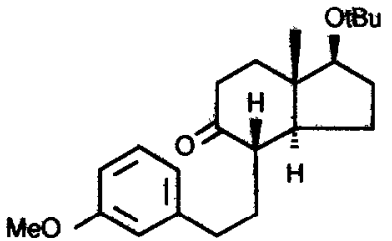

$10 \mathrm{~d}$

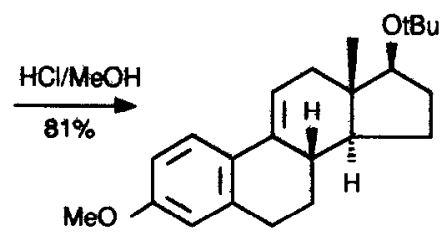

11

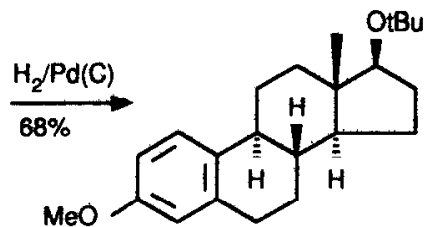

12

The seco-sterordal ketone 10d was converted into the unsaturated sterold 11 by Fnedel-Crafts cyclization with dry hydrochlonc acid in methanol at $0^{\circ} \mathrm{C} 9,10$ Subsequent hydrogenation with palladium on charcoal in ethyl acetate at room temperature yielded the title compound 12 The optical rotations of 11 and of 12 were in agreement with the values reported by Wiechert et al ${ }^{9}$ and Cohen et al 10 The 3,17 - protected estrone denvative 12 can be easily converted by known methodology into pharmaceutically important 19 -norsteroids such as norethindrone 11

Startıng from the $\mathrm{CD}$ bulding block 5 , which was prepared enantiomencally pure in a $S$-proline catalyzed asymmetric aldol condensation according to the procedure reported by Eder, Sauer and Wiechert ${ }^{12}$ and by Hajos and Parrish, ${ }^{13}$ the title compound 12 was synthesized in 5 steps in an overall yleld of $24 \%$ This synthesis opens a very short and efficient route towards estrone and other 19 norsteroids and uses easily available and inexpensıve startıng matenals 


\section{EXPERIMENTAL}

NMR spectra were taken on Varian VXR 200, 500 and XL 200 spectrometers IR spectra were taken on a Perkın Elmer Mod 298 spectrometer Mass spectra were recorded on Varian MAT 731 and 311 A spectrometers Optıcal rotations were measured on a Perkın Elmer Mod 141 polarimeter TLC analyses were performed on Polygram Sil G/UV 254 silica gel plates Silica gel 60 (240-400 mesh) from E Merck Darmstadt was used for flash chromatography Combustion analyses were carned out by the microanalytical laboratory of the University of Gottıngen All reactions were carned out under dry and oxygen-free argon $\mathrm{CuBr}_{\mathrm{SMe}}$ was recrystallized from dimethyl sulfide/pentane and dried under argon All reagents and solvents were dried and purified before using THF, HMPT and the $12 \mathrm{M}$ DIBAH solution in toluene were degassed by the freeze-pumping method The starting enone 5 was prepared according to the described procedure 12,13 or afforded by the Schening AG The phenylsulfides, used for the synthesis of the $\alpha$-chlorophenylsulfides 8a-d were prepared from the corresponding mesylates or halides by $\mathrm{S}_{\mathrm{N}}$ 2-replacement with thiophenol/ $\mathrm{K}_{2} \mathrm{CO}_{3}$ in acetone

Preparation of the aluminium enolates 2 and 6, general procedure: To a slurry of $\mathrm{CuBr} \mathrm{SMe}_{2}(205$ $\mathrm{mg}, 1 \mathrm{mmol})$ in THF (10 ml) a tert -butyllithium solution (1 7M in pertane, $065 \mathrm{ml}, 11 \mathrm{mmol})$ was added at $-50^{\circ} \mathrm{C}$ and sturred for 15 min Hexamethylphosphonc triamide (HMPA) ( $716 \mathrm{mg}, 4 \mathrm{mmol}$ ) was added to the solution and cooled down to $-100^{\circ} \mathrm{C}$ A mixture of disobutylaluminium hydnde (DIBAH) (1 $2 \mathrm{M}$ in toluene, $125 \mathrm{ml}, 15 \mathrm{mmol}$ ) and HMPA (716 $\mathrm{mg}, 4 \mathrm{mmol}$ ) was slowly added durng $10 \mathrm{~min} \mathrm{~A}$ solution of $1(1 \mathrm{mmol}, 96 \mathrm{mg})$ or $5(1 \mathrm{mmol}, 222 \mathrm{mg})$ in THF $(1 \mathrm{ml})$ was added dropwise during 15 min The temperature was allowed to rise up to $-80^{\circ} \mathrm{C}$ and the reaction mixture was stirred for $2 \mathrm{~h}$ During two further hours the temperature was allowed to warm up to $-40^{\circ} \mathrm{C}$

Preparation of the $\alpha$-chloroalkylphenylsulfides 8a-d, general procedure: To a slurry of $\mathrm{N}$ chlorosuccinimide $(147 \mathrm{~g}, 110 \mathrm{mmol})$ in $\mathrm{CCl}_{4}(20 \mathrm{ml})$ the alkylphenylsulfide $(10 \mathrm{mmol})$ was added dropwise at $-2{ }^{\circ} \mathrm{C}$ and the mixture was stırred for $16 \mathrm{~h}$ at this temperature The solution was separated by filtration from succinimide, the solvent was evaporated and the crude products 8a-d were used without further purification

trans-3-Methyl-2-(1'-phenylthioethyl)cyclopentanone (4): The enolate 2 was prepared from $\mathrm{CuBrSMe}_{2}$ (377 mg, $184 \mathrm{mmol}$ ), tert -butyllthium (1 7M in pentane, $113 \mathrm{ml}, 192 \mathrm{mmol}$ ), HMPA (2 $54 \mathrm{~g}, 142 \mathrm{mmol}$ ), DIBAH (1 2M in toluene, $83 \mathrm{ml}, 10 \mathrm{mmol}$ ) and 3-methyl-2-cyclopenten-1-one (1) (077 g, $8 \mathrm{mmol}$ ) A $\mathrm{ZnCl}_{2}$ solution (1 OM in ether, $25 \mathrm{ml}, 25 \mathrm{mmol}$ ) was added at $-40^{\circ} \mathrm{C}$ and sturring was continued for $30 \mathrm{~min}$ Finally $\alpha$-chloroethylphenylsulfide (172 $\mathrm{g}, 10 \mathrm{mmol}$ ) was added dropwise and the reaction mixture was stirred for $4 \mathrm{~h}$ at $-20^{\circ} \mathrm{C}$ Within $8 \mathrm{~h}$ the solution was allowed to warm up to roomtemp, extracted five times with $\mathrm{Et}_{2} \mathrm{O}(50 \mathrm{ml}$ each time) and the combined organic phases were washed with $1 \mathrm{M} \mathrm{HCl}(40 \mathrm{ml})$, saturated aqueous $\mathrm{NaHCO}_{3}(40 \mathrm{ml})$ and 3 tumes with $\mathrm{H}_{2} \mathrm{O}(20 \mathrm{ml}$ each tume) The organic phase was dried over $\mathrm{MgSO}_{4}$ and the solvent evaporated in vacuo Chromatography on silica gel with ether/pentane 14 afforded 4 ( $126 \mathrm{~g}, 54 \mathrm{mmol}, 67 \%)$ as a pale yellow oll $\left(R_{f}=029\right.$ and 0 36) - ratio of diastereomers 4411 - IR (neat): $v=3040(\mathrm{C}-\mathrm{H} /$ phenyl), $1730(\mathrm{C}=\mathrm{O}), 1580$ $\left(\mathrm{C}=\mathrm{C} /\right.$ phenyl), 735 and $685 \mathrm{~cm}^{-1}\left(\mathrm{C}-\mathrm{H} /\right.$ monosub phenyl) - $\left.{ }^{1} \mathrm{H} \mathrm{NMR} \mathrm{(200} \mathbf{M H z}, \mathrm{CDCl}_{3}\right): \delta=085-$ $250(\mathrm{~m}, 12 \mathrm{H}),, 387\left(\mathrm{dq}, \mathrm{J}=25 \mathrm{~Hz}\right.$ and $\left.7 \mathrm{~Hz}, 1 \mathrm{H}, \mathrm{C}_{1}-\mathrm{H}\right), 715-750\left(\mathrm{~m}, 5 \mathrm{H}_{2}-\mathrm{SC}_{6} \mathrm{H}_{5}\right)-{ }^{13} \mathrm{C} \mathrm{NMR}$ $\left(50 \mathrm{MHz}, \mathrm{CDCl}_{3}\right): \delta=1770(2062)\left(\mathrm{C}_{1}-\mathrm{CH}_{3}\right), 2071$ (21 08) $\left(\mathrm{C}_{3}-\mathrm{CH}_{3}\right), 2943$ (29 71) (C-4), 3331 (34 45) (C-3), 3844 (38 50) (C-5), 4182 (43 93) (C-2), 5998 (61 32) (C'-1), 12663 (126 93), 12892 (128 78) and 13095 (131 95) (C-H/phenyl), 13568 (135 32) (C/phenyl), 21800 (218 31) (C-1) signals in brakets for the mınor diastereomer - MS $(70 \mathrm{eV}):(\mathrm{m} / \mathrm{z})=55\left(100 \%, \mathrm{C}_{3} \mathrm{H}_{3} \mathrm{O}^{+}\right), 97\left(80 \%, \mathrm{M}^{+}\right.$- 
$\left.\mathrm{SC}_{6} \mathrm{H}_{5}-\mathrm{C}_{2} \mathrm{H}_{4}\right), 125\left(80 \%, \mathrm{M}^{+}-\mathrm{SC}_{6} \mathrm{H}_{5}\right), 234\left(95 \%, \mathrm{M}^{+}\right)$- HRMS (70 eV): calculated for $\mathrm{C}_{13} \mathrm{H}_{18} \mathrm{OS}$ 2341078 , found 2341078

[1S,3aS,7aS]-1-tert-Butoxy-2,3,3a,6,7,7a-hexahydro-7a-methyl-5-trimethylsiloxy-1H-indene (7): The enolate 6 was prepared according to the general procedure from $\mathrm{CuBr}_{\mathrm{SMe}}(062 \mathrm{~g}, 3 \mathrm{mmol})$, tertbutyllthuum (1 $88 \mathrm{ml}, 32 \mathrm{mmol}$ ), HMPA (423 g, $24 \mathrm{mmol}$ ), DIBAH (3 $75 \mathrm{ml}, 45 \mathrm{mmol}$ ) and 5 (0 67 $\mathrm{g}, 3 \mathrm{mmol})$ To the reaction mixture chlorotrimethylsilane $(065 \mathrm{~g}, 6 \mathrm{mmol})$ was added at $-40^{\circ} \mathrm{C}$ After stırning for $3 \mathrm{~h}$ thethylamine ( $242 \mathrm{~g}, 24 \mathrm{mmol}$ ) was added at this temperature and the reaction mixture was allowed to warm up to room temp and was extracted with pentane $(200 \mathrm{ml})$ in a perforator for $4 \mathrm{~h}$ After solvent evaporation and silica gel chromatography (ether/pentane $14, R_{\mathrm{f}}=0$ 74) 7 (068 g, 76\%) was obtained as colourless oll that solidified when refrigerated, $[\alpha]_{\mathbf{D}^{20}}{ }^{20}+23.2^{\circ}\left(\mathrm{c}=10, \mathrm{CHCl}_{3}\right)$ IR (neat): $v=1650 \mathrm{~cm}^{-1}$ (C=C/olefine) - $\left.{ }^{1} \mathrm{H} \mathrm{NMR} \mathrm{(200} \mathrm{MHz,CDCl}\right): \delta=014\left(\mathrm{~s}, 9 \mathrm{H}, \mathrm{Si}_{1}\left(\mathrm{CH}_{3}\right)_{3}\right)$, 075 - 250 (m, 9H, CH, CH 3$), 076\left(\mathrm{~s}, 3 \mathrm{H}, \mathrm{C}_{7 \mathrm{a}}-\mathrm{CH}_{3}\right), 108\left(\mathrm{~s}, 9 \mathrm{H}, \mathrm{C}\left(\mathrm{CH}_{3}\right)_{3}\right), 341\left(\mathrm{t}, \mathrm{J}=8 \mathrm{~Hz}, 1 \mathrm{H}, \mathrm{C}_{1^{-}}\right.$ $\mathrm{H}), 470\left(\mathrm{~d}, \mathrm{~J}=4 \mathrm{~Hz}, 1 \mathrm{H}, \mathrm{C}_{4}-\mathrm{H}\right)-{ }^{13} \mathrm{C} \mathrm{NMR}\left(50 \mathrm{MHz}, \mathrm{CDCl}_{3}\right): \delta=020\left(\mathrm{~S}_{1}\left(\mathrm{CH}_{3}\right)_{3}\right), 1074\left(\mathrm{C}_{7 \mathrm{a}^{-}}\right.$

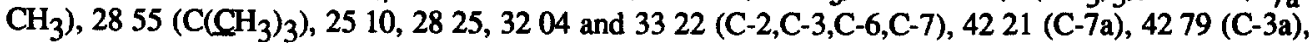
$7191\left(\mathrm{C}_{\left.\left(\mathrm{CH}_{3}\right)_{3}\right), 78} 80(\mathrm{C}-1), 10486(\mathrm{C}-4), 15020(\mathrm{C}-5)-\mathrm{MS}(70 \mathrm{eV}):(\mathrm{m} / \mathrm{z})=57\left(100 \%, \mathrm{C}_{4} \mathrm{H}_{9}{ }^{+}\right)\right.$, $73\left(85 \%, \mathrm{~S}_{1}\left(\mathrm{CH}_{3}\right)_{3}{ }^{+}\right), 296\left(8 \%, \mathrm{M}^{+}\right)-\mathrm{C}_{17} \mathrm{H}_{32} \mathrm{O}_{2} \mathrm{Si}(296.5)$ calc C,68 86, H,10 88, found C,69 06, $\mathrm{H}, 1102 \%$

Thioalkylation of the silylenol ether 7 , general procedure: The slylenol ether $7(030 \mathrm{~g}, 1 \mathrm{mmol})$ was dissolved in $\mathrm{CH}_{2} \mathrm{Cl}_{2}(4 \mathrm{ml})$, cooled to $-78{ }^{\circ} \mathrm{C}$ and $11 \mathrm{mmol}$ of the $\alpha$-chloroalkylphenylsulfides 8 were added dropwise $A$ catalytic amount of $\mathrm{ZnCl}_{2}(2-25 \mathrm{~mol} \%)$ was added and the muxture was stirred for $05 \mathrm{~h}$ at $-78{ }^{\circ} \mathrm{C}$ and was allowed to warm up to $-20^{\circ} \mathrm{C}$ within $4 \mathrm{~h}$ A saturated $\mathrm{NaHCO}_{3}$ solution $(25 \mathrm{ml})$ was added, the aqueous phase was extracted twice with ether $(20 \mathrm{ml}$ each tume) and the combined organic phases were dried over $\mathrm{MgSO}_{4}$ After the solvent was removed in vacuo the crude product 9 was purfied by chromatography on silica gel

(1S,1'RS,3aS,4RS,7aS)-1-tert-Butoxy-4-(1'-phenylthioethyl)-7a-methyl-3a,4,7,7a-tetrahydro-5(6)indan-5-one (9a): $\alpha$-Chloroethylphenylsulfide $8 \mathrm{a}$ (1 $566 \mathrm{~g}, 91 \mathrm{mmol}, 91 \%)$ was prepared from $\mathrm{N}$ chlorosuccinimide (147 g, $11 \mathrm{mmol}$ ) and ethylphenylsulfide (138 $\mathrm{g}, 10 \mathrm{mmol}$ ) The $\beta$ phenylthioketone $9 \mathrm{a}(225 \mathrm{mg}, 062 \mathrm{mmol}, 71 \%)$ was prepared according to the general procedure from the silylenol ether 7 (260 mg, $088 \mathrm{mmol})$, the $\alpha$-Chloro-ethylphenylsulfide $8 \mathrm{a}(167 \mathrm{mg}, 097 \mathrm{mmol})$ and $\mathrm{ZnCl}_{2}(10 \mathrm{M}$ in ether, $002 \mathrm{ml}, 002 \mathrm{mmol})$, purified by chromatography on $150 \mathrm{~g}$ silica gel with ether/pentane/tnethylamine 16001 and obtained a an colourless oll $\left(R_{f}=039\right.$ and 033 for four dastereomers) - ratio of diastereomers 5221 - IR (neat): $v=3050$ and 3030 (C-H/phenyl), 1695 $(\mathrm{C}=\mathrm{O}), 1570 \mathrm{~cm}^{-1}(\mathrm{C}=\mathrm{C} / \mathrm{phenyl})$ - $\mathbf{1}_{\mathrm{H}} \mathrm{NMR}\left(200 \mathrm{MHz}_{\mathrm{CDCl}}\right): \delta=098\left(\mathrm{~s}, 3 \mathrm{H}, \mathrm{C}_{7 \mathrm{a}}-\mathrm{CH}_{3}\right), 118(\mathrm{~s}$, $\left.9 \mathrm{H}, \mathrm{C}\left(\mathrm{CH}_{3}\right)_{3}\right), 138\left(\mathrm{~d}, \mathrm{~J}=7 \mathrm{~Hz}, 3 \mathrm{H}, \mathrm{CH}_{3} \mathrm{CHSPh}\right), 0$ 75-2 $60\left(\mathrm{~m}, 9 \mathrm{H}, \mathrm{CH}\right.$ and $\left.\mathrm{CH}_{2}\right), 264$ (dd, J = 29 and $\left.128 \mathrm{~Hz}, 1 \mathrm{H}, \mathrm{C}_{4}-\mathrm{H}\right), 349\left(\mathrm{dd}, \mathrm{J}=75 \mathrm{~Hz}\right.$ and $\left.85 \mathrm{~Hz}, 1 \mathrm{H}, \mathrm{C}_{1}-\mathrm{H}\right), 362(\mathrm{dq}, \mathrm{J}=29 \mathrm{~Hz}$ and $7 \mathrm{~Hz}$, $1 \mathrm{H}, \mathrm{CH}-\mathrm{SPh}), 714-752\left(\mathrm{~m}, 5 \mathrm{H}, \mathrm{SC}_{6} \mathrm{H}_{5}\right)-{ }^{13} \mathrm{C} \mathrm{NMR}\left(50 \mathrm{MHz}_{3} \mathrm{CDCl}_{3}\right): \delta=1127\left(\mathrm{C}_{7 \mathrm{a}}-\mathrm{CH}_{3}\right), 1895$ $\left(\mathrm{CH}_{3}-\mathrm{CH}-\mathrm{SPh}\right), 2868\left(\mathrm{O}-\mathrm{C}\left(\mathrm{CH}_{3}\right)_{3}\right), 2538,3189,3541,3800$ (C-2, C-3, C-6, C-7), 4299 (C-7a), 4206 and 4650 (C-3a and C-1'), 5611 (C-4), 7258 (C-9), 12641 (C-4/phenyl), 12893 (C-2/phenyl), 13064 (C-3/phenyl), 13696 (C-1/phenyl), 21062 (C-5), only signals for the major diastereomer - MS $(70 \mathrm{eV}):(\mathrm{m} / \mathrm{z})=57\left(100 \%, \mathrm{C}_{4} \mathrm{H}_{9}+\right), 194\left(60 \%, \mathrm{M}^{+}-\mathrm{SPh}-\mathrm{C}_{4} \mathrm{H}_{9}{ }^{+}\right), 360\left(24 \%, \mathrm{M}^{+}\right)-$HRMS (70 eV): calculated for $\mathrm{C}_{22} \mathrm{H}_{32} \mathrm{O}_{2} \mathrm{~S} 3602123$, found $3602123-\mathrm{C}_{22} \mathrm{H}_{32} \mathrm{O}_{2} \mathrm{~S}$ (360 6) calc C,73 29, H,8 95, found $\mathrm{C}, 7445, \mathrm{H}, 911 \%$

(1S,3aS,4S,7aS)-1-tert-Butoxy-4-(1'-methyl-1'-phenylthioethyl)-7a-methyl-3a,4,7,7a-tetrahydro5(6)-Indan-5-one (9b) : $\alpha$-Chloro- $\alpha$-methylethylphenylsulfide $8 \mathrm{~b}(315 \mathrm{mg}, 169 \mathrm{mmol}, 85 \%$ ) was 
prepared from N-chlorosuccinimide (300 mg, $2.25 \mathrm{mmol}$ ) and iso-propylphenylsulfide ( $305 \mathrm{mg}, 20$ mmol) According to the general procedure $\beta$-phenylthioketone $9 b$ was prepared from the silylenol ether 7 (260 mg, $088 \mathrm{mmol}$ ), $\alpha$-chloro- $\alpha$-methylethylphenylsulfide $8 \mathrm{~b}\left(180 \mathrm{mg}, 097 \mathrm{mmol}\right.$ ) and $\mathrm{ZnCl}_{2}$ (1.0 M in ether, $002 \mathrm{ml}, 0.02 \mathrm{mmol}$ ) After punfication by flash chromatography on $28 \mathrm{~g}$ silica gel (desactivated with $3 \mathrm{~g} \mathrm{H}_{2} \mathrm{O}$ ) with ether/pentane/triethylamine $1200019 \mathrm{~b}$ (197 mg, $053 \mathrm{mmol}, 60 \%$ ) was obtained as a colourless solid $\left(R_{\mathrm{f}}=017\right)-\mathrm{d} \mathrm{e}>95 \%-\mathrm{m.p} .78-81^{\circ} \mathrm{C}-$ IR (nujol): $v=3050$ (C-H/phenyl), $1685(\mathrm{C}=\mathrm{O}), 740$ and $690 \mathrm{~cm}^{-1}(\mathrm{C}-\mathrm{H} /$ monosub phenyl) - $1 \mathrm{H}$ NMR (200 MHz,CDCl$)$ : $\delta=091\left(\mathrm{~s}, 3 \mathrm{H}, \mathrm{C}_{7 \mathrm{a}}-\mathrm{CH}_{3}\right), 115\left(\mathrm{~s}, 9 \mathrm{H}, \mathrm{C}\left(\mathrm{CH}_{3}\right)_{3}\right), 130\left(\mathrm{~s}, 6 \mathrm{H}, \mathrm{PhS}-\mathrm{C}\left(\mathrm{CH}_{3}\right)_{2}\right), 080-263(\mathrm{~m}, 11 \mathrm{H} ; \mathrm{CH}$ and $\left.\mathrm{CH}_{2}\right), 351$ (dd, $\mathrm{J}=8 \mathrm{~Hz}$ and $\left.85 \mathrm{~Hz}, \mathrm{C}_{1}-\mathrm{H}\right), 720-756\left(\mathrm{~m}, 5 \mathrm{H}, \mathrm{SC}_{6} \mathrm{H}_{5}\right)-{ }^{13} \mathrm{C}$ NMR (50

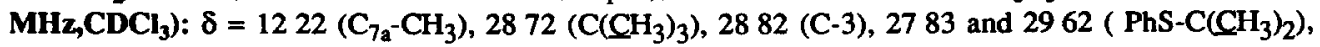
3203 (C-7), 3472 (C-2), 3886 (C-6), 4366 (C-7a), 4697 and 5101 (C-3a and C-1'), 6133 (C-4), $7254\left(\mathrm{C}\left(\mathrm{CH}_{3}\right)_{3}\right), 7910(\mathrm{C}-1), 12849$ (C-2/phenyl), 12888 (C-4/phenyl), 131,43 (C-1/phenyl), 13795 (C-3/phenyl), 21379 (C-5) - MS (70 eV) : (m/z) = $57\left(59 \%, \mathrm{C}_{4} \mathrm{H}_{9}{ }^{+}\right), 209\left(100 \%, \mathrm{M}^{+}-\mathrm{C}_{4} \mathrm{H}_{9}{ }^{+}-\mathrm{SPh}\right)$, $265\left(30 \%, \mathrm{M}^{+}-\mathrm{SPh}\right), 374\left(24 \%, \mathrm{M}^{+}\right)$, - HRMS (70 eV): calculated for $\mathrm{C}_{23} \mathrm{H}_{34} \mathrm{O}_{2} \mathrm{~S} 3742280$, found $3742279-\mathrm{C}_{23} \mathrm{H}_{34} \mathrm{O}_{2} \mathrm{~S}(374.6)$ calc C,73 75, H,9 15, found C,73 74, H,9 01\%

\section{(1S,1'RS,3aS,4RS,7aS)-1-tert-Butoxy-4-(2'-phenyl-1'-phenylthıethyl)-7a-methyl-3a,4,7,7a-}

tetrahydro-5(6)-indan-5-one (9c) : The $\alpha$-chloro- $\beta$-phenylethylphenylsulfide $8 \mathrm{c}$ (425 mg, $171 \mathrm{mmol}$, $85 \%$ ) was prepared from $\mathrm{N}$-chlorosuccinımıde $(294 \mathrm{mg}, 22 \mathrm{mmol}$ ) and B-phenylethylphenylsulfide $(429 \mathrm{mg}, 20 \mathrm{mmol})$ The thioalkylation of silylenol ether 7 (200 mg, $068 \mathrm{mmol})$ with $8 \mathrm{c}(195 \mathrm{mg}, 074$ mmol) and $\mathrm{ZnCl}_{2}(10 \mathrm{M}$ in ether, $003 \mathrm{ml}, 003 \mathrm{ml}$ ) according to the general procedure yielded the $\beta$ thiophenylketone $9 \mathrm{c}(165 \mathrm{mg}, 038 \mathrm{mmol}, 56 \%)$ After chromatography on $65 \mathrm{~g}$ silica gel (desactivated with $6 \mathrm{~g} \mathrm{H}_{2} \mathrm{O}$ ) with ether/pentane/tnethylamine $160019 \mathrm{c}$ was obtained as a colourless oll $\left(R_{\mathrm{f}}=040\right.$ and 036 for four diastereomers) - ratıo of diastereomers 8411 - IR (neat): $v=3040$ (C-H/phenyl), $1695(\mathrm{C}=\mathrm{O}), 1595$ and $1575\left(\mathrm{C}=\mathrm{C} /\right.$ phenyl), 740 and $690 \mathrm{~cm}^{-1}(\mathrm{C}-\mathrm{H} /$ monosub phenyl) $-1 \mathrm{H} \mathrm{NMR} \mathrm{(200}$ $\left.\mathrm{MHz}_{2} \mathrm{CDCl}_{3}\right): \delta=087\left(\mathrm{~s}, 3 \mathrm{H}, \mathrm{C}_{7 \mathrm{a}}-\mathrm{CH}_{3}\right), 112\left(\mathrm{~s}, 9 \mathrm{H}, \mathrm{C}\left(\mathrm{CH}_{3}\right)_{3}\right), 266(\mathrm{dd}, \mathrm{J}=3 \mathrm{~Hz}$ and $13 \mathrm{~Hz}, 1 \mathrm{H}$, $\left.\mathrm{C}_{4}-\mathrm{H}\right), 085-252$ and $300-340\left(\mathrm{~m}, 12 \mathrm{H}, \mathrm{CH}\right.$ and $\left.\mathrm{CH}_{2}\right), 346\left(\mathrm{dd}, \mathrm{J}=75 \mathrm{~Hz}\right.$ and $\left.85 \mathrm{~Hz}, 1 \mathrm{H}, \mathrm{C}_{1}-\mathrm{H}\right)$, $710-738\left(\mathrm{~m}, 10 \mathrm{H}\right.$, phenyl-H) - $\left.{ }^{13} \mathrm{C} \mathrm{NMR}\left(50 \mathrm{MHz}_{2} \mathrm{CDCl}_{3}\right): \delta=1111\left(\mathrm{C}_{7 \mathrm{a}}-\mathrm{CH}_{3}\right), 2865\left(\mathrm{C}_{3} \mathrm{CH}_{3}\right)_{3}\right)$, $2466,3168,3529,3823,4092$ and 4280 (C-2,C-3,C-6,C-7,C-7a,C-2'), 4687 and 5115 (C-3a and C1'), 5371 (C-4), 7256 (C-9), 7926 (C-1), 12637 and 12641 (C-4/phenyl), 128 39, 128 79, 12926 and 13072 (C-2,C-3/phenyl), 13761 and 13984 (C-1/phenyl), 21091 (C-5), only signals for the major diastereomer - MS (70 eV): $(\mathrm{m} / \mathrm{z})=57\left(88 \%, \mathrm{C}_{4} \mathrm{H}_{9}+\right), 91\left(100 \%, \mathrm{CH}_{2}-\mathrm{Ph}\right), 270\left(41 \%, \mathrm{M}^{+}-\mathrm{SPh}\right.$ $\left.\mathrm{C}_{4} \mathrm{H}_{9}{ }^{+}\right), 327\left(20 \%, \mathrm{M}^{+}-\mathrm{SPh}\right), 426\left(50 \%, \mathrm{M}^{+}\right)$- HRMS (70 eV): calculated for $\mathrm{C}_{28} \mathrm{H}_{36} \mathrm{O}_{2} \mathrm{~S} 4362436$, found $4362436-\mathrm{C}_{28} \mathrm{H}_{36} \mathrm{O}_{2} \mathrm{~S}(436,7)$ calc $\mathrm{C}, 7702, \mathrm{H}, 831$, found $\mathrm{C}, 7808, \mathrm{H}, 847 \%$

(1S,1'RS,3aS,4RS,7aS)-1-tert-Butoxy-4-(2'-m-methoxyphenyl-1'-phenylthioethyl)-7a-methyl-

3a,4,7,7a-tetrahydro5(6)-indan-5-one (9d) : $\alpha$-Chloro- $\beta$-(m-methoxyphenyl)ethylphenylsulfide 8d (797 $\mathrm{mg}, 286 \mathrm{mmol}, 95 \%$ ) was prepared by chlorination of $\beta$-(m-methoxyphenyl)ethylphenylsulfide (733 mg, $30 \mathrm{mmol}$ ) with $\mathrm{N}$-chlorosuccinımide $(440 \mathrm{mg}, 33 \mathrm{mmol}$ ) According to the general procedure the $\beta$-phenylthioketone 9d was prepared from the silylenol ether $7(250 \mathrm{mg}, 085 \mathrm{mmol}), 8 \mathrm{~d}(259 \mathrm{mg}$, $093 \mathrm{mmol}$ ) and $\mathrm{ZnCl}_{2}(10 \mathrm{M}$ in ether, $02 \mathrm{ml}, 02 \mathrm{mmol}$ ) Purification by flash chromatography on $29 \mathrm{~g}$ silica gel (desactivated with $3 \mathrm{~g} \mathrm{H}_{2} \mathrm{O}$ ) with ether/pentane/tnethylamine 16001 yielded $9 \mathrm{~d}(256 \mathrm{mg}$, $055 \mathrm{mmol}, 65 \%$ ) as a colourless oil with $R_{\mathrm{f}}=022$ and 027 - ratio of diastereomers 12421 - IR (neat): $v=3050\left(\mathrm{C}-\mathrm{H} /\right.$ phenyl), $1705(\mathrm{C}=\mathrm{O}), 1595$ and $1575(\mathrm{C}=\mathrm{C} / \mathrm{phenyl}), 775$ and $745 \mathrm{~cm}^{-1}(\mathrm{C}$ H/disubs phenyl) - 1H NMR (200 $\left.\mathrm{MHz}_{2} \mathrm{CDCl}_{3}\right): \delta=088\left(\mathrm{~s}, 3 \mathrm{H}, \mathrm{C}_{7 \mathrm{a}}-\mathrm{CH}_{3}\right), 113\left(\mathrm{~s}, 9 \mathrm{H}, \mathrm{C}\left(\mathrm{CH}_{3}\right)_{3}\right)$, 268 (dd, $\mathrm{J}=22 \mathrm{~Hz}$ and $13 \mathrm{~Hz}, 1 \mathrm{H}, \mathrm{C}_{4}-\mathrm{H}$ ), $080-260$ and $265-319\left(\mathrm{~m}, 11 \mathrm{H}, \mathrm{CH}\right.$ and $\mathrm{CH}_{2}$ ), 336 (td, $\mathrm{J}=22 \mathrm{~Hz}$ and $\left.8 \mathrm{~Hz}, 1 \mathrm{H}, \mathrm{C}_{1}-\mathrm{H}\right), 347\left(\mathrm{dd}, \mathrm{J}=72 \mathrm{~Hz}\right.$ and $\left.80 \mathrm{~Hz}, 1 \mathrm{H}, \mathrm{C}_{1}-\mathrm{H}\right), 376\left(\mathrm{~s}, 3 \mathrm{H}, \mathrm{OCH}_{3}\right), 662$ - $681\left(\mathrm{~m}, 3 \mathrm{H}\right.$, phenyl-CH), $709-739\left(\mathrm{~m}, 6 \mathrm{H},-\mathrm{SC}_{6} \mathrm{H}_{5}\right.$ and $\mathrm{C}_{5}$-phenyl) ${ }^{13} \mathrm{C} \mathrm{NMR}\left(50 \mathrm{MHz}, \mathrm{CDCl}_{3}\right)$ 


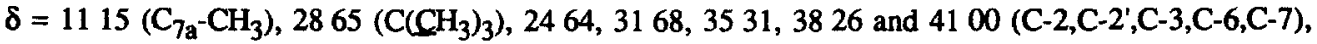
4280 (C-7a), 4690 and $\left.5087\left(\mathrm{C}-1^{\prime}, \mathrm{C}-3 \mathrm{a}\right), 5364(\mathrm{C}-4), 5517\left(\mathrm{OCH}_{3}\right), 7257\left(\mathrm{C}_{(\mathrm{CH}}\right)_{3}\right), 7926(\mathrm{C}-1)$, $11186,11487,12160,12636,12879,12934,13071,13760,141$ 39, 159 62, (C-phenyl), 21089 (C5), signals for the major diastereomer. - MS $(70 \mathrm{eV}):(\mathrm{m} / \mathrm{z})=57\left(100 \%, \mathrm{C}_{4} \mathrm{H}_{9}^{+}\right), 357\left(10 \%, \mathrm{M}^{+}-\mathrm{SPh}\right)$, $466\left(20 \%, \mathrm{M}^{+}\right)$- HRMS (70 eV): calculated for $\mathrm{C}_{29} \mathrm{H}_{38} \mathrm{O}_{3} \mathrm{~S} 466.2541$ found $4662542-\mathrm{C}_{29} \mathrm{H}_{38} \mathrm{O}_{3} \mathrm{~S}$ (466.7) calc C,74 64, H,8 21, found C,74 66, H,8 37\%

Reductive desulfurization of 9, general procedure: $5 \mathrm{~g}$ Raney nickel were washed 10 tumes with 10 ml $96 \%$ ethanol , 5 more times with $10 \mathrm{ml}$ dry ethanol and suspended in $10 \mathrm{ml}$ dry ethanol A solution of $\beta$-phenylthoketones $9 \mathrm{a}$-d $(05 \mathrm{mmol})$ in $8 \mathrm{ml}$ ethanol was added at roomtemp After $3 \mathrm{~h}$ the Raney nickel was removed by filtration and washed 5 times with $15 \mathrm{ml} \mathrm{Et}_{2} \mathrm{O}$ After evaporation of the solvent the crude ketones 10a-d were punfied by chromatography

(1S,3aS,4S,7aS)-(+)-1-tert-Butoxy-4-ethyl-7a-methyl-3a,4,7,7a-tetrahydro-5(6)-indan-5-one (10a) : The desulfurization was carried out according to the general procedure with $\beta$-phenylthioketone $9 \mathrm{a}$ (150 $\mathrm{mg}, 042 \mathrm{mmol}$ ) and Raney nickel ( $2 \mathrm{~g}$ ) After purification by flash chromatography with ether/pentane - 16 the ketone $10 \mathrm{a}(98 \mathrm{mg}, 039 \mathrm{mmol}, 93 \%)$ was obtained as a colourless solid $\left(R_{\mathrm{f}}=052\right)-\mathrm{d} \mathrm{e}>$ 95\% $-[\alpha]_{\mathrm{D}}{ }^{20}=+4875^{\circ}\left(\mathrm{c}=08, \mathrm{CHCl}_{3}\right)-$ m.p. $44-46^{\circ} \mathrm{C}-$ IR (neat): $v=1695 \mathrm{~cm}^{-1}(\mathrm{C}=\mathrm{O})-{ }^{1} \mathrm{H}$ NMR (200 MHz, CDCl $): \delta=088\left(\mathrm{t}, \mathrm{J}=73 \mathrm{~Hz}, 3 \mathrm{H}, \mathrm{CH}_{2}-\mathrm{CH}_{3}\right), 103\left(\mathrm{~s}, 3 \mathrm{H}, \mathrm{C}_{7 \mathrm{a}}-\mathrm{CH}_{3}\right), 114(\mathrm{~s}, 9 \mathrm{H}$, $\left.\mathrm{C}\left(\mathrm{CH}_{3}\right)_{3}\right), 075-258\left(\mathrm{~m}, 12 \mathrm{H}, \mathrm{CH}\right.$ and $\left.\mathrm{CH}_{2}\right), 345\left(\mathrm{dd}, \mathrm{J}=74 \mathrm{~Hz}\right.$ and $\left.88 \mathrm{~Hz}, 1 \mathrm{H}, \mathrm{C}_{1}-\mathrm{H}\right)-{ }^{13} \mathrm{C}$ NMR (50 $\left.\mathbf{M H z}, \mathrm{CDCl}_{3}\right): \delta=1113\left(\mathrm{CH}_{2}-\mathrm{CH}_{3}\right), 1148\left(\mathrm{C}_{7 \mathrm{a}}-\mathrm{CH}_{3}\right), 1928\left(\mathrm{CH}_{2}-\mathrm{CH}_{3}\right), 2453$ (C-3), 28.66

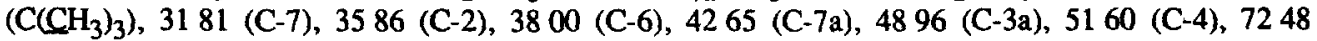

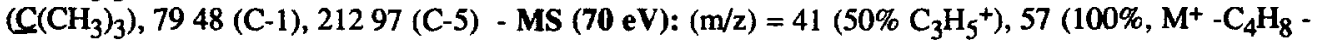
$\left.\mathrm{C}_{2} \mathrm{H}_{4}\right), 196\left(40 \%, \mathrm{M}^{+}-\mathrm{C}_{4} \mathrm{H}_{8}\right), 252\left(23 \%, \mathrm{M}^{+}\right)$- HRMS (70 eV): calculated for $\mathrm{C}_{16} \mathrm{H}_{28} \mathrm{O}_{2} 2522089$, found $2522089-\mathrm{C}_{16} \mathrm{H}_{28} \mathrm{O}_{2}$ (252.4) calc C,76 14, H,11 18, found C,76 17, H,11 26\%

(1S,3aS,4S,7aS)-(+)-1-tert-Butoxy-4-iso-propyl-7a-methyl-3a,4,7,7a-tetrahydro-5(6)-indan-5-one (10b) : According to the general procedure 10b was prepared from $9 \mathrm{~b}(80 \mathrm{mg}, 022 \mathrm{mmol})$ by treatment with Raney nickel $(15 \mathrm{~g})$ The ketone $10 \mathrm{~b}(51 \mathrm{mg}, 019 \mathrm{mmol}, 90 \%)$ was obtained after flash chromatography on $27 \mathrm{~g}$ silica gel with ether/pentane 120 as a colourless solid $\left(R_{\mathrm{f}}=020\right)-\mathrm{de}>$ 95\% . $[\alpha]_{\mathrm{p}^{20}}=+701^{\circ}\left(\mathrm{c}=09, \mathrm{CHCl}_{3}\right)-$ m.p. $34^{\circ} \mathrm{C}-$ IR (nujol): $\mathrm{v}=1695 \mathrm{~cm}^{-1}(\mathrm{C}=\mathrm{O})-1 \mathrm{H} \mathrm{NMR}$ $\left(200 \mathrm{MHz}_{2} \mathrm{CDCl}_{3}\right): \delta=092\left(\mathrm{~s}, 3 \mathrm{H}, \mathrm{C}_{7 \mathrm{a}}-\mathrm{CH}_{3}\right), 097\left(\mathrm{~d}, \mathrm{~J}=7 \mathrm{~Hz}, 6 \mathrm{H}, \mathrm{CH}\left(\mathrm{CH}_{3}\right)_{2}\right), 114(\mathrm{~s}, 9 \mathrm{H}$, $\left.\mathrm{C}\left(\mathrm{CH}_{3}\right)_{3}\right), 085-253\left(\mathrm{~m}, 11 \mathrm{H}, \mathrm{CH}\right.$ and $\left.\mathrm{CH}_{2}\right), 345\left(\mathrm{dd}, \mathrm{J}=8 \mathrm{~Hz}\right.$ and $\left.85 \mathrm{~Hz}, 1 \mathrm{H}, \mathrm{C}_{1}-\mathrm{H}\right)-{ }^{13} \mathrm{C} \mathrm{NMR}$

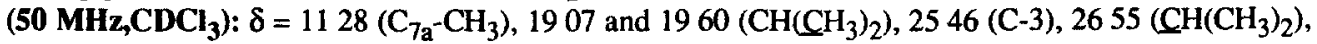

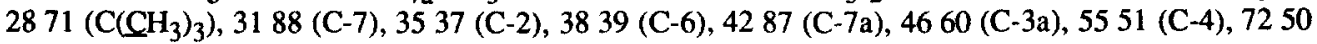
$\left(\mathrm{C}\left(\mathrm{CH}_{3}\right)_{3}\right), 7944(\mathrm{C}-1), 21283(\mathrm{C}-5)-\mathrm{MS}(70 \mathrm{eV}):(\mathrm{m} / \mathrm{z})=57\left(100 \%, \mathrm{C}_{4} \mathrm{H}_{9}^{+}\right), 167\left(14 \%, \mathrm{M}^{+}-\mathrm{C}_{4} \mathrm{H}_{8}\right.$ $\left.-\mathrm{C}_{3} \mathrm{H}_{7}\right), 195\left(40 \%, \mathrm{M}^{+}-\mathrm{C}_{4} \mathrm{H}_{8}-\mathrm{CH}_{3}\right), 210\left(48 \%, \mathrm{M}^{+}-\mathrm{C}_{4} \mathrm{H}_{8}\right), 266\left(34 \%, \mathrm{M}^{+}\right)$- HRMS (70 eV): calculated for $\mathrm{C}_{17} \mathrm{H}_{30} \mathrm{O}_{2} 2662246$, found $2662245-\mathrm{C}_{17} \mathrm{H}_{30} \mathrm{O}_{2}$ (266.4) calc C,76 64, H,11 35, found C,76 52, H,1131\%

(1S,3aS,4S,7aS)-(+)-1-tert-Butoxy-4-(2'-phenylethyl)-7a-methyl-3a,4,7,7a-tetrahydro-5(6)-indan-5one (10c) : Treatment of $9 \mathrm{c}(132 \mathrm{mg}, 030 \mathrm{mmol})$ with Raney nickel $(2 \mathrm{~g})$ afforded after purfication by flash chromatography on $29 \mathrm{~g}$ silica gel with ether/pentane 16 the ketone $10 \mathrm{c}(88 \mathrm{mg}, 027 \mathrm{mmol}$, $88 \%)$ as an colourless solid $\left(R_{\mathrm{f}}=042\right) \cdot \mathrm{de}>95 \%-[\alpha]_{\mathrm{D}}{ }^{20}=+2842^{\circ}\left(\mathrm{c}=10, \mathrm{CHCl}_{3}\right)-$ m.p. : $70^{\circ} \mathrm{C}$ - IR (nujol): $v=3040$ (C-H/phenyl), $1695(\mathrm{C}=\mathrm{O}), 740$ and $690 \mathrm{~cm}^{-1}(\mathrm{C}-\mathrm{H} /$ monosubs phenyl) ${ }_{1} \mathbf{H}$ NMR (200 MHz, $\left.\mathrm{CDCl}_{3}\right): \delta=102\left(\mathrm{~s}, 3 \mathrm{H}, \mathrm{C}_{7 \mathrm{a}}-\mathrm{CH}_{3}\right), 113\left(\mathrm{~s}, 9 \mathrm{H}, \mathrm{C}\left(\mathrm{CH}_{3}\right)_{3}\right), 080-206(\mathrm{~m}, 9 \mathrm{H}$, $\mathrm{CH}$ and $\mathrm{CH}_{2}$ ), $225-258\left(\mathrm{~m}, 4 \mathrm{H}, \mathrm{CH}_{2}-\mathrm{CH}_{2}-\mathrm{Ph}\right), 273$ (ddd, $\mathrm{J}=52 \mathrm{~Hz}, 108 \mathrm{~Hz}$ and $134 \mathrm{~Hz}, 1 \mathrm{H}, \mathrm{C}_{4^{-}}$ $\mathrm{H}), 345\left(\mathrm{dd}, \mathrm{J}=76 \mathrm{~Hz}\right.$ and $\left.86 \mathrm{~Hz}, 1 \mathrm{H}, \mathrm{C}_{1}-\mathrm{H}\right), 711-732\left(\mathrm{~m}, 5 \mathrm{H}\right.$, phenyl) $-{ }^{13} \mathrm{C}$ NMR (50 


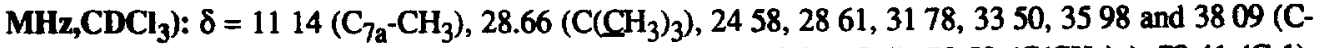
2,C-3,C-6,C-7,C-1' and C-2'), 4281 (C-7a), 4969 (C-3a), $5005(\mathrm{C}-4), 7252\left(\mathrm{C}^{\prime}\left(\mathrm{CH}_{3}\right)_{3}\right), 79.41(\mathrm{C}-1)$, 12566 (C-4/phenyl), 12827 and 12838 (C-2 and C-3/phenyl), 14281 (C-1/phenyl), 212.80 (C-5) MS (70 eV): $(\mathrm{m} / \mathrm{z})=57\left(100 \%, \mathrm{C}_{4} \mathrm{H}_{9}{ }^{+}\right), 167\left(93 \%, \mathrm{M}^{+}-\mathrm{C}_{4} \mathrm{H}_{9}{ }^{+}-\mathrm{CH}_{2} \mathrm{Ph}\right), 181\left(85 \%, \mathrm{M}^{+}-\mathrm{C}_{4} \mathrm{H}_{9}{ }^{+}-\right.$ $\left.\mathrm{CH}_{2} \mathrm{CH}_{2} \mathrm{Ph}\right), 272\left(4 \%, \mathrm{M}^{+}-\mathrm{C}_{4} \mathrm{H}_{9}^{+}\right), 329\left(4 \% \mathrm{M}^{+}\right)$- HRMS (70 eV): calculated for $\mathrm{C}_{22} \mathrm{H}_{33} \mathrm{O}_{2}$ 3282402 , found $3282402-\mathrm{C}_{22} \mathrm{H}_{33} \mathrm{O}_{2}$ (328.5) calc C,80 44, H,9 82, found, C,80 72, H,9 87\%

\section{(1S,3aS,4S,7aS)-(+)-1-tert-Butoxy-4-(2'-m-methoxyphenylethyl)-7a-methyl-3a,4,7,7a-tetrahydro-}

5(6)-indan-5-one (10d) : The seco-steroid 10d9,10 was obtained as a colourless oll (130 mg, 036 mmol, 97\%, $\left.R_{f}=028\right)$ from the $\beta$-phenylthioketone $9 \mathrm{~d}(175 \mathrm{mg}=0375 \mathrm{mmol})$ and Raney nickel $(3 \mathrm{~g})$ after purification by flash chromatography on $29 \mathrm{~g}$ silica gel with ether/pentane 16 diastereomencally and enantiomencally pure $[\alpha]_{\mathrm{D}}^{20}=+3163^{\circ}\left(\mathrm{c}=10, \mathrm{CHCl}_{3}\right)$, lit ${ }^{10}[\alpha]_{\mathrm{D}}^{25}=+2746^{\circ}(\mathrm{c}=10$, $\left.\mathrm{CHCl}_{3}\right)$ - IR (neat): $\mathrm{v}=3040\left(\mathrm{C}-\mathrm{H} /\right.$ phenyl), $1705(\mathrm{C}=\mathrm{O}), 1595$ and $1575\left(\mathrm{C}=\mathrm{C} /\right.$ phenyl), $775 \mathrm{~cm}^{-1}(\mathrm{C}-$ $\mathrm{H} / 1$ 3-disubs phenyl) - 1H NMR (200 $\left.\mathrm{MHz} \mathrm{CDCl}_{3}\right): \delta=102\left(\mathrm{~s}, 3 \mathrm{H}, \mathrm{C}_{7 \mathrm{a}}-\mathrm{CH}_{3}\right), 114$ (s, 9H, $\left.\mathrm{C}\left(\mathrm{CH}_{3}\right)_{3}\right), 080-263\left(\mathrm{~m}, 13 \mathrm{H}, \mathrm{C}_{3 \mathrm{a}}-\mathrm{H}\right.$ and $\left.\mathrm{CH}_{2}\right), 271$ (ddd, $\mathrm{J}=52 \mathrm{~Hz}, 108 \mathrm{~Hz}$ and $134 \mathrm{~Hz} ; 1 \mathrm{H}, \mathrm{C}_{4}^{-}$ $\mathrm{H}), 345\left(\mathrm{dd}, \mathrm{J}=75 \mathrm{~Hz}\right.$ and $\left.85 \mathrm{~Hz}, 1 \mathrm{H}, \mathrm{C}_{1}-\mathrm{H}\right), 380\left(\mathrm{~s}, 3 \mathrm{H},-\mathrm{OCH}_{3}\right), 667-686(\mathrm{~m}, 3 \mathrm{H}, \mathrm{CH} / \mathrm{phenyl})$, $719\left(\mathrm{dt},{ }^{3} \mathrm{~J}_{\text {ortho }}=76 \mathrm{~Hz},{ }^{5} \mathrm{~J}_{\text {para }}=09 \mathrm{~Hz}, 1 \mathrm{H}, \mathrm{C}_{5}-\mathrm{H} / \mathrm{phenyl}\right)-{ }^{13} \mathrm{C} \mathrm{NMR}\left(50 \mathrm{MHz}, \mathbf{C D C l}_{3}\right): \delta=1117$

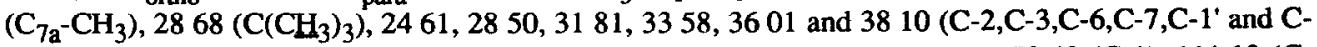

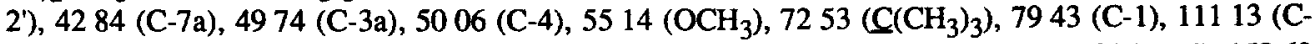
6/phenyl), 11404 (C-2/phenyl), 12083 (C-4/phenyl), 12923 (C-5/phenyl), 14448 (C-3/phenyl), 15962 (C-1/phenyl), $21285(\mathrm{C}-5)-\mathrm{MS}(70 \mathrm{eV}):(\mathrm{m} / \mathrm{z})=57\left(100 \%, \mathrm{C}_{4} \mathrm{H}_{9}{ }^{+}\right), 167\left(82 \%, \mathrm{M}^{+}-\mathrm{C}_{4} \mathrm{H}_{8}-\mathrm{CH}_{3} \mathrm{O}-\right.$ $\left.\mathrm{C}_{6} \mathrm{H}_{4}-\mathrm{C}_{2} \mathrm{H}_{4}^{+}\right), 181\left(40 \%, \mathrm{M}^{+}-\mathrm{CH}_{3} \mathrm{O}-\mathrm{C}_{6} \mathrm{H}_{4}-\mathrm{CH}_{2}{ }^{+}\right), 301\left(4 \%, \mathrm{M}^{+}-\mathrm{C}_{4} \mathrm{H}_{9}{ }^{+}\right), 358\left(23 \%, \mathrm{M}^{+}\right)-$HRMS $(70 \mathrm{eV})$ : calculated for $\mathrm{C}_{23} \mathrm{H}_{34} \mathrm{O}_{3} 3582508$, found 3582507

(8S,13S,14S,17S)-(+)-17-tert-Butoxy-3-methoxyestra-1,3,5(10),9(11)-tetraene (11): The seco-stero1d $10 d(80 \mathrm{mg}, 022 \mathrm{mmol})$ was dissolved at $0^{\circ} \mathrm{C}$ in $\mathrm{MeOH}(2 \mathrm{ml})$ and $10 \mathrm{~N} \mathrm{HCl}(02 \mathrm{ml})$ was added The solution was stirred for $4 \mathrm{~h}$ at $0^{\circ} \mathrm{C}$, allowed to warm up to roomtemp and stirred for additional $4 \mathrm{~h}$ The mixture was kept overnight at $-28^{\circ} \mathrm{C}$ to complete the crystallization The crude product was separated by filtration and recrystallization from $\mathrm{MeOH}$ afforded the unsaturated steroid $119,10(60 \mathrm{mg}, 018$ mmol, $81 \%)$ as a colourless solıd $-[\alpha]_{D^{20}}{ }^{20}=+1078^{\circ}\left(\mathrm{c}=10, \mathrm{CHCl}_{3}\right)$, lit ${ }^{9}[\alpha]_{\mathrm{D}}{ }^{\mathrm{RT}}=+1023^{\circ}(\mathrm{c}=$ $\left.05, \mathrm{CHCl}_{3}\right)$, lit $10[\alpha]_{\mathrm{D}^{25}}=+10127^{\circ}\left(\mathrm{c}=10, \mathrm{CHCl}_{3}\right)$ - m.p. $129^{\circ} \mathrm{C}$, lit ${ }^{9}$ m.p $131-133^{\circ} \mathrm{C}$, lit ${ }^{10}$ m p $133-134^{\circ} \mathrm{C}-$ IR (nujol): $v=1625(\mathrm{C}=\mathrm{C} /$ olefine $), 1605$ and $1585 \mathrm{~cm}^{-1}(\mathrm{C}=\mathrm{C} /$ phenyl) $-1 \mathrm{H} \mathrm{NMR}$ $\left(200 \mathrm{MHz}_{3} \mathrm{CDCl}_{3}\right): \delta=078\left(\mathrm{~s}, 3 \mathrm{H}, \mathrm{C}_{13}-\mathrm{CH}_{3}\right), 117\left(\mathrm{~s}, 9 \mathrm{H}, \mathrm{C}\left(\mathrm{CH}_{3}\right)_{3}\right), 121-225(\mathrm{~m}, 10 \mathrm{H}, \mathrm{CH}$ and $\left.\mathrm{CH}_{2}\right), 275-298\left(\mathrm{~m}, 2 \mathrm{H}, \mathrm{C}_{6}-\mathrm{H}\right), 354\left(\mathrm{dd}, \mathrm{J}=75 \mathrm{~Hz}\right.$ and $\left.85 \mathrm{~Hz}, 1 \mathrm{H}, \mathrm{C}_{17}-\mathrm{H}\right), 378\left(\mathrm{~s}, 3 \mathrm{H}, \mathrm{OCH}_{3}\right)$, $612\left(\mathrm{~m}, 1 \mathrm{H}, \mathrm{C}_{11}-\mathrm{H}\right), 659\left(\mathrm{~d},{ }^{4} \mathrm{~J}_{\text {meta }}=3 \mathrm{~Hz}, 1 \mathrm{H}, \mathrm{C}_{4}-\mathrm{H}\right), 671\left(\mathrm{dd},{ }^{3} \mathrm{~J}_{\text {ortho }}=8 \mathrm{~Hz},{ }^{4} \mathrm{~J}_{\text {meta }}=3 \mathrm{~Hz}, 1 \mathrm{H}\right.$, $\left.\mathrm{C}_{2}-\mathrm{H}\right), 754\left(\mathrm{~d},{ }^{3} \mathrm{~J}_{\text {ortho }}=8 \mathrm{~Hz}, 1 \mathrm{H}, \mathrm{C}_{1}-\mathrm{H}\right)-{ }^{13} \mathrm{C} \mathrm{NMR}\left(50 \mathrm{MHz}, \mathrm{CDCl}_{3}\right): \delta=1168\left(\mathrm{C}_{13}-\mathrm{CH}_{3}\right), 2880$ $\left(\mathrm{C}\left(\mathrm{CH}_{3}\right)_{3}\right), 2442,2826,3021$, and $3128(\mathrm{C}-7, \mathrm{C}-12, \mathrm{C}-15, \mathrm{C}-16), 3899(\mathrm{C}-8), 3958(\mathrm{C}-6), 4117(\mathrm{C}-$ 13), $4738(\mathrm{C}-14), 5521\left(\mathrm{OCH}_{3}\right), 7225\left(\mathrm{C}\left(\mathrm{CH}_{3}\right)_{3}\right), 8082$ (C-17), $11260(\mathrm{C}-2), 11325$ (C-4), 11797 (C-11), 12509 (C-1), 12764 (C-9), $13499(\mathrm{C}-10), 13748$ (C-5), $15822(\mathrm{C}-3)-\mathrm{MS}$ (70 eV): (m/z) = $57\left(44 \%, \mathrm{C}_{4} \mathrm{H}_{9}{ }^{+}\right), 267\left(30 \%, \mathrm{M}^{+}-\mathrm{OC}\left(\mathrm{CH}_{3}\right)_{3}{ }^{+}\right), 283\left(45 \%, \mathrm{M}^{+}-\mathrm{C}_{4} \mathrm{H}_{9}{ }^{+}\right), 340\left(100 \%, \mathrm{M}^{+}\right)-$HRMS (70 eV): calculated for $\mathrm{C}_{23} \mathrm{H}_{32} \mathrm{O}_{2} 3402402$, found $3402402-\mathrm{C}_{23} \mathrm{H}_{32} \mathrm{O}_{2}(340,5)$ calc $\mathrm{C}, 8113, \mathrm{H}, 947$, found $\mathrm{C}, 8121, \mathrm{H}, 950 \%$

(8S,9S,13S,14S,17S)-(+)-17-tert-Butoxy-3-methoxyestra-1,3,5(10)-triene (12) : A mixture of 11 (34 $\mathrm{mg}, 010 \mathrm{mmol}$ ), palladium (5 mg, 10\% on carbon) and ethyl acetate (12 ml) was stirred under hydrogen for $3 \mathrm{~h}$ The catalyst was filtered off with suction on Celite and the Celite was washed with ethyl acetate $(20 \mathrm{ml})$ The ethyl acetate was evaporated in vacuo and the crude product purfied by flash chromatography on $5 \mathrm{~g}$ silica gel with ether/pentane 16 to yield $129,10,14(23 \mathrm{mg}, 68 \%)$ as a 
colourless solid $\left(R_{\mathrm{f}}=053\right)-[\alpha]_{\mathrm{D}}^{20}=+63.3^{\circ}\left(\mathrm{c}=0.9, \mathrm{CHCl}_{3}\right)$, lit ${ }^{10}[\alpha]_{\mathrm{D}}{ }^{25}=+6220^{\circ}(\mathrm{c}=1.0$, $\mathrm{CHCl}_{3}$ ) - m.p. 89-91 ${ }^{\circ} \mathrm{C}$; lit. 10 m.p 90-92 - IR (nujol): $v=1605$ and $1580 \mathrm{~cm}-1$ (C=C/phenyl) - $1 \mathrm{H}$ $\operatorname{NMR}\left(500 \mathrm{MHz}, \mathrm{CDCl}_{3}\right): \delta=075\left(\mathrm{~s}, 3 \mathrm{H}, \mathrm{C}_{13}-\mathrm{CH}_{3}\right), 115\left(\mathrm{~s}, 9 \mathrm{H} ; \mathrm{C}\left(\mathrm{CH}_{3}\right)_{3}\right), 0.80-220\left(\mathrm{~m}, 11 \mathrm{H}, \mathrm{CH}_{2}\right.$ and $\mathrm{C}_{8}-\mathrm{H}$ ), 2.18 (ddd, $\mathrm{J}=4 \mathrm{~Hz}, 115 \mathrm{~Hz}$ and $115 \mathrm{~Hz}, 1 \mathrm{H}, \mathrm{C}_{9}-\mathrm{H}$ ), 228 (ddd, $\mathrm{J}=4 \mathrm{~Hz}, 7 \mathrm{~Hz}$ and $13 \mathrm{~Hz}$, $1 \mathrm{H}, \mathrm{C}_{14}-\mathrm{H}$ ), 285 (dd, $\mathrm{J}=75 \mathrm{~Hz}$ and $8 \mathrm{~Hz}, 2 \mathrm{H}, \mathrm{C}_{6}-\mathrm{H}$ ), 3.45 (dd, $\mathrm{J}=75 \mathrm{~Hz}$ and $8 \mathrm{~Hz}, 1 \mathrm{H}, \mathrm{C}_{17}-\mathrm{H}$ ), 378 $\left(\mathrm{s}, 3 \mathrm{H}, \mathrm{OCH}_{3}\right), 6.63\left(\mathrm{~d},{ }^{4} \mathrm{~J}_{\text {meta }}=3 \mathrm{~Hz}, 1 \mathrm{H}, \mathrm{C}_{4}-\mathrm{H}\right), 671\left(\mathrm{dd},{ }^{4} \mathrm{~J}_{\text {meta }}=3 \mathrm{~Hz},{ }^{3} \mathrm{~J}_{\text {ortho }}=9 \mathrm{~Hz}, 1 \mathrm{H} ; \mathrm{C}_{2}-\mathrm{H}\right)$, $722\left(\mathrm{~d}, 3 \mathrm{~J}_{\text {ortho }}=9 \mathrm{~Hz}, 1 \mathrm{H}, \mathrm{C}_{1}-\mathrm{H}\right)-{ }^{13} \mathrm{C} \mathrm{NMR}\left(50 \mathrm{MHz}, \mathrm{CDCl}_{3}\right): \delta=1162\left(\mathrm{C}_{13}-\mathrm{CH}_{3}\right), 2877$ $\left(\mathrm{C}\left(\mathrm{CH}_{3}\right)_{3}\right), 23.50,2640,2729,29.91,31.23$ and 37.24 (C-6,C-7,C-11,C-12,C-15,C-16), 4274 (C-13),

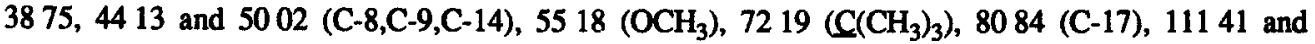
113.74 (C-2,C-4), 126.35 (C-1), 13290 (C-10), 13806 (C-5), 15736 (C-3) - MS (70 eV): (m/z) = 57 $\left(100 \%, \mathrm{C}_{4} \mathrm{H}_{9}{ }^{+}\right), 286\left(68 \%, \mathrm{M}^{+}-\mathrm{C}_{4} \mathrm{H}_{8}\right), 342\left(80 \%, \mathrm{M}^{+}\right)$- HRMS (70 eV) calculated for $\mathrm{C}_{23} \mathrm{H}_{34} \mathrm{O}_{2}$ 3422559 , found 3422559

Acknowledgement - Financial support by the Deutsche Forschungsgemeinschaft and the Stuftung Volkswagenwerk is gratefully acknowledged We thank Dr G Sauer of the Schenng AG, Berlin, for providing valuable startıng materials

\section{REFERENCES}

1 a) G Quinkert, H Stark, Angew Chem 1983, 95, 651, Angew Chem Intl Ed Engl 1983, 22, 637,

b) R Wiechert, Angew Chem 1977, 89, 513, Angew Chem.Intl Ed Engl 1977, 16, 506,

c) R Wiechert, Angew Chem 1970, 82, 331, Angew Chem.Intl Ed Engl 1970, 9, 321

2 A R Daniewsk1, J Kiegiel, Synth Comm 1988, 18, 115

3. a) T Tsuda, H Satom1, T Hayash1, T Kawamoto, T Saegusa, $J$ Org Chem 1986, 51, 537,

b) T.Tsuda, H Satom, T.Hayashı, T Saegusa, $J$ Org Chem 1987, 52, 439

4 a) A R Daniewska, J Kiegiel, E Piotrowska, T Warchol, W Wojciechowska, Liebigs Ann Chem 1988, 593, b) A R Daniewsk1, J Kiegiel, J Org Chem 1988, 53, 5535, A R Daniewsk1, M R Uskokovic, Tetrahedron Lett 1990, 5599

5 A R Daniewsk1, E Plotrowska, W Wojciechowska, Liebigs Ann Chem 1989, 1061

6 U Groth, T Huhn, N Richter, Liebigs Ann Chem, submitted

7 a) I Paterson, Tetrahedron 1988, 44, 4207, b) I Paterson, I Fleming, Tetrahedron Lett 1979, 995, c) I Paterson, I Fleming, Tetrahedron Lett 1979, 2179

8 T Mukhopadhyay, D Seebach, Helv Chim Acta 1982, 39, 385

9 U Eder, H Gibian, G Haffer, G Neef, G Sauer, R Wiechert, Chem Ber 1976, 40, 681

10 N Cohen, B L Banner, W F Eichel, D R Parnsh, G Saucy, J Org Chem 1975, 40, 681

11 G Quinkert, U Schwartz, H Stark, W D Weber, F Adam, H Bater, G Frank, G Durner, Liebigs Ann Chem 1982, 1999

12 U Eder, G Sauer, R Wiechert, Angew Chem 1971, 83, 492, Angew Chem Int Ed Engl 1971, 10,496

13 a) Z G Hajos, D R Parnsh, $J$ Org Chem, 1974, 39, 1615, b) R A Michel1, Z G Hajos, N Cohen, D R Parnsh, L A Portland, W Sciamanna, M A Scott, P A Wehrl, J Org Chem, $1975,40,675$

14 T Kametan, H Matsumoto, H Nemoto, K Fukumoto, J Am Chem Soc, 1978, 100, 6218 